\title{
The overexpression of FKBP4 in patients with lung adenocarcinoma predicts poor prognosis and tumor progression
}

\section{Sha Tian}

Hunan University of Chinese Medicine

\section{Shang qing Wang}

The Affiliated Hospital of Xuzhou Medical University: Xuzhou Medical University Affiliated Hospital

Piao Zheng

Hunan University of TCM: Henan University of Traditional Chinese Medicine

\section{Xu Zhu}

Hunan University of TCM: Henan University of Traditional Chinese Medicine

\section{Huan Han}

Kunming hospital of traditional chinese medicine

\section{Xiao di Huang}

Hunan University of TCM: Henan University of Traditional Chinese Medicine

\section{Jing Li}

The First Hospital of Hunan university of chinese medicine

\section{Xuefei Tian ( $\nabla 003640 @$ hnucm.edu.cn )}

Tianjin Hospital of Integrated Traditional Chinese and Western Medicine https://orcid.org/0000-00028407-2268

\section{Primary research}

Keywords: fkbp4, luad, expression, analysis, high, tumor, gene, cohort

Posted Date: August 3rd, 2021

DOl: https://doi.org/10.21203/rs.3.rs-733351/v1

License: (c) (i) This work is licensed under a Creative Commons Attribution 4.0 International License. Read Full License 


\section{The overexpression of $F K B P 4$ in patients with lung adenocarcinoma predicts poor prognosis and tumor progression}

1 Sha Tian', Shangqing Wang ${ }^{2}$, Piao Zheng ${ }^{1}$, Xu Zhu ${ }^{1}$, Huan Han ${ }^{3}$, Xiaodi Huang ${ }^{4}$, Jing Li ${ }^{5}$ and

2 Xuefei Tian ${ }^{1 *}$

$3 \quad{ }^{1}$ Department of Internal Medicine, College of Integrated Chinese and Western Medicine of Hunan

4 University of Chinese Medicine, Changsha, China

$5 \quad{ }^{2}$ Department of Hepatobiliary and Pancreatic Surgery, Affiliated Hospital of Xuzhou Medical 6 University, Xuzhou, China

$7 \quad{ }^{3}$ Department of Pediatrics, Kunming Hospital of Traditional Chinese Medicine, Kunming, China

$8{ }^{4}$ Hunan Key Laboratory of Traditional Chinese Medicine Prescription and Syndromes Translational

9 Medicine, Hunan University of Chinese Medicine, Changsha, China

$10{ }^{5}$ Department of Oncology, The First Hospital of Hunan University of Chinese Medicine, Changsha, 11 China

$12 *$ Correspondence:

13 Xuefei Tian

14 003640@hnucm.edu.cn

15 Keywords: lung adenocarcinoma, FKBP4, TCGA, bioinformatics analysis, GSEA, prognosis.

16 Abstract

17 Background: The FK506-binding protein 4 (FKBP4), a tumor-related gene, plays a vital role in 18 tumorigenesis and cancer progression. The study is aimed to clarify the effect of $F K B P 4$ in lung 19 adenocarcinoma (LUAD).

20 Methods: Relying on The Cancer Genome Atlas (TCGA) cohort, the FKBP4 expression difference between LUAD tissues and non-tumor tissues was first detected, and verified with public tissue microarrays (TMAs), clinical LUAD specimen cohort and Gene Expression Omnibus (GEO) cohort. Then, logistic regression analysis and chi-square test were applied to detect the correlation between FKBP4 expression and clinicopathological parameters. Kaplan-Meier survival analysis and Cox regression model were utilized to evaluate the effect of FKBP4 expression on survival. Signaling pathways related to LUAD were obtained via employing Gene Set Enrichment Analysis (GSEA).

Results: The FKBP4 expression level in LUAD samples was dramatically higher than that in nontumor samples. High FKBP4 expression in LUAD is associated with gender, pathological stage, T classification, lymph node metastasis and distant metastasis. The Kaplan-Meier curve indicated a poor prognosis for LUAD patients with high $F K B P 4$ expression. Multivariate analysis suggested that the high FKBP4 expression was a vital independent predictor of poor overall survival (OS). GSEA showed that a total of 15 signaling pathways were enriched in samples with high FKBP4 expression phenotype. 
33 Conclusions: FKBP4 may be an oncogene in LUAD, and is promised to become a prognostic indicator

34 and therapeutic target for LUAD.

\section{Background}

Lung cancer is the cancer with the highest cancer incidence and mortality in the world [1]. The 5-year survival rate is less than 20\% [2]. Among non-small cell lung cancer (NSCLC), lung adenocarcinoma (LUAD) is the most common histological subtype, accounting for about $40 \%$ of all types of lung malignancies [3]. During the last decade, researchers across the world have carried out large-scale genomic studies to reveal some driver genes of LUAD. It has been reported that the most common somatic mutations in lung cancer include TP53, KRAS, KEAP1, STK11, and EGFR [4-6]. Although some progress has been made in lung cancer research, there are still a large number of LUAD patients who do not have effective targeted treatment options, either because of the lack of known gene mutations in key oncogenic signaling pathways, or because it is difficult to target oncogenic mutations. The purpose of this study is to reveal a potential diagnostic and therapeutic target for patients with LUAD.

The FK506-binding protein 4 (FKBP4), a member of immunophilin family, has been proved to play a key role in immune regulation, protein folding, and transportation [7]. Recent studies have revealed that FKBP4 might play an important role in tumorigenesis and progression of various cancers, and is expected to become an effective biomarker [8]. For instance, Alain et al. [9] found that the abnormal expression of $F K B P 4$ has a huge effect on the breast cancer progression and prognosis. In particular, FKBP4 depletion can decrease cell growth and proliferation in triple-negative breast cancer cell models and xenograft tumor models. Meng et al. [10] proved that FKBP4 is highly expressed in NSCLC, accelerating the malignant progression of NSCLC by activating the Akt/mTOR signaling pathway.

However, few studies have explored the association between FKBP4 and LUAD, especially in terms of prognostic biomarkers. Based on public databases and our own fresh frozen tissue specimen cohort, this study has investigated the associations between the FKBP4 expression level and clinicopathological characteristics of LUAD, as well as the prognostic significance of $F K B P 4$, in order to provide more evidences for the potential role of FKBP4 in LUAD. In addition, GSEA was implemented to deepen the understandings of the signal pathways involved in $F K B P 4$ regulatory networks related to LUAD.

\section{Materials and Methods}

\subsection{TCGA cohort and fresh frozen tissue specimen cohort}

The TCGA cohort including the raw gene expression data for 497 LUAD tissues and 54 adjacent nontumor tissues were obtained from the TCGA database (https://portal.gdc.cancer.gov). Similarly, the corresponding clinical data of LUAD patients containing the information of age, gender, pathological stage, T stage, $\mathrm{N}$ stage, $\mathrm{M}$ stage, and vital status (Table 1) were also received. This study is in full compliance with the guidelines of the National Institute of Health (NIH) TCGA human subject protection and data access policies. Besides, we have collected 51 pairs of fresh frozen LUAD tissues and adjacent non-tumor tissues at the Affiliated Hospital of Xuzhou Medical University. These samples were preserved at $-80^{\circ} \mathrm{C}$ for quantitative real-time PCR (qRT-PCR). This project was granted approval by the Ethics Committee of the Affiliated Hospital of Xuzhou Medical University.

TABLE 1 | Statistics of the TCGA cohort related to patients with LUAD 


\begin{tabular}{|c|c|c|c|}
\hline Characteristics & Variable & Patients (486) & Percentages (\%) \\
\hline \multirow[t]{3}{*}{ Age } & $<65$ years & 209 & 43.00 \\
\hline & $\geq 65$ years & 258 & 53.09 \\
\hline & unknown & 19 & 3.91 \\
\hline \multirow[t]{2}{*}{ Gender } & Male & 222 & 45.68 \\
\hline & Female & 264 & 54.32 \\
\hline \multirow[t]{5}{*}{ Pathological stage } & I & 262 & 53.91 \\
\hline & II & 112 & 23.05 \\
\hline & III & 79 & 16.25 \\
\hline & IV & 25 & 5.14 \\
\hline & unknown & 8 & 1.65 \\
\hline \multirow[t]{5}{*}{$\mathrm{T}$ classification } & $\mathrm{T} 1$ & 163 & 33.54 \\
\hline & $\mathrm{T} 2$ & 260 & 53.50 \\
\hline & T3 & 41 & 8.44 \\
\hline & T4 & 19 & 3.91 \\
\hline & TX & 3 & 0.61 \\
\hline \multirow[t]{5}{*}{$\mathrm{N}$ classification } & N0 & 312 & 64.20 \\
\hline & N1 & 90 & 18.52 \\
\hline & $\mathrm{N} 2$ & 70 & 14.40 \\
\hline & N3 & 2 & 0.41 \\
\hline & NX & 12 & 2.47 \\
\hline \multirow[t]{3}{*}{ M classification } & M0 & 333 & 68.52 \\
\hline & M1 & 24 & 4.94 \\
\hline & MX & 129 & 26.54 \\
\hline \multirow[t]{2}{*}{ Vital status } & Alive & 324 & 66.67 \\
\hline & Death & 162 & 33.33 \\
\hline
\end{tabular}

\section{$75 \quad 2.2 \quad F K B P 4$ expression and survival analysis}

76 First, the online public UALCAN (http://ualcan.path.uab.edu/) was utilized to observe the mRNA and

77 protein levels of $F K B P 4$ in human pan-cancer. Then, the raw mRNA expression data from the TCGA

78 cohort was preprocessed via employing the Perl programming including data sorting, data merging and

79 identification conversion. Based on the limma package and beeswarm package, we visualize FKBP4

80 expression data through drawing the scatter difference chart and paired difference chart. Following, we

81 extracted survival data of LUAD patients matching FKBP4 expression data from the clinical data, and

82 delete samples without survival time or survival status information. As a result, we got data on 455

83 LUAD patients who met the requirements of the research. More, depending on the median value of

$84 \quad F K B P 4$ expression, the 455 LUAD patients were divided into two groups (high FKBP4 expression 85 group and low FKBP4 expression group). Relying on data in the two groups, we utilized survival

86 package to draw the Kaplan-Meier survival curve.

\section{$87 \quad 2.3 \quad$ RNA extraction and qRT-PCR}

88 According to instructions, we utilized Trizol reagent (Invitrogen) to extract total RNA from fresh 89 frozen LUAD tissues and adjacent non-tumor tissues. Then, Trans Script one-step guide DNA removal 90 and complementary DNA synthesis super mix were used for the reverse transcription reaction. The 91 primer sequences for PCR amplification were as follows: FKBP4, forward: 5'92 ATTGCCATAGCCACCATGAAG-3', reverse: 5'- CCTGCTGAACCGTAGGCATATT-3'.

\section{$93 \quad 2.4 \quad$ The verification of $F K B P 4$ by GEO and human protein atlas}

94 In GEO database (https://www.ncbi.nlm.nih.gov/geo), "lung adenocarcinoma" was considered as the 95 search term, and "Homo sapiens" was set as the qualifier to filter out available microarrays for the 
verification of FKBP4 expression in LUAD. By filtering out the datasets with small sample size $(\mathrm{N}<50)$, we obtained 6 reliable datasets (GSE101929, GSE11969, GSE18842, GSE27262, GSE32863 and GSE75037), including 338 LUAD tissue samples and 250 non-tumor tissue samples, as well as the FKBP4 expression value of each sample (Table 2). Relying on Review Manage 5.3 software, we implemented the meta-analysis to validate the differences of FKBP4 expression level between LUAD samples and non-tumor samples. Based on standard mean difference (SMD) with a 95\% confidence interval (CI), we calculated the combined value. Chi-square $\left(\chi^{2}\right)$ and $I^{2}$ statistical tests were utilized to evaluate the heterogeneity between the six screened datasets. If $p>0.05$ or $I^{2}<50 \%$, a fixed effect model was chosen to calculate the combined effect, otherwise a random effect model was selected $(p<0.05$ or $\left.I^{2}>50 \%\right)$. Moreover, taking "FKBP4" and "lung adenocarcinoma" as search terms, we also obtained immunohistochemical results of patients with LUAD from the human protein atlas database (http://www.proteinatlas.org).

TABLE 2 | Information of screened GEO microarrays related to LUAD

\begin{tabular}{|c|c|c|c|c|c|}
\hline GEO datasets & Year & Country & Platform & Sample & $\mathbf{N}$ \\
\hline \multirow[t]{2}{*}{ GSE101929 } & 2019 & USA & GPL570 & LUAD & 32 \\
\hline & & & & Non-LUAD & 34 \\
\hline \multirow[t]{2}{*}{ GSE11969 } & 2013 & Japan & GPL7015 & LUAD & 94 \\
\hline & & & & Non-LUAD & 5 \\
\hline \multirow[t]{2}{*}{ GSE18842 } & 2019 & Spain & GPL570 & LUAD & 46 \\
\hline & & & & Non-LUAD & 45 \\
\hline \multirow[t]{2}{*}{ GSE27262 } & 2019 & China & GPL570 & LUAD & 25 \\
\hline & & & & Non-LUAD & 25 \\
\hline \multirow[t]{2}{*}{ GSE32863 } & 2019 & USA & GPL6884 & LUAD & 58 \\
\hline & & & & Non-LUAD & 58 \\
\hline \multirow[t]{2}{*}{ GSE75037 } & 2019 & USA & GPL6884 & LUAD & 83 \\
\hline & & & & Non-LUAD & 83 \\
\hline
\end{tabular}

\subsection{Univariate and multivariate cox regression analyses}

In order to quantitatively assess the independent predictive effect of different clinical pathological factors and FKBP4 expression on survival, the hazard ratio (HR) and 95\% CI were calculated, and the Cox regression model was used for univariate and multivariate analyses. The independent prognostic effect of FKBP4 on survival was assessed by adjusting for confounding factors. Specifically, Perl was applied to preprocess the clinical data in the TCGA cohort, and remove samples with incomplete clinical information. After matching the processed complete clinical data with the FKBP4 expression data, the LUAD patients were divided into the high FKBP4 expression group or the low FKBP4 expression group according to the median $F K B P 4$ expression value. In the end, depending on complete data of 316 patients with LUAD, we carried out univariate and multivariate cox regression analyses.

\subsection{Gene Set Enrichment Analysis (GSEA)}

Here, GSEA was applied to confirm whether a given set of genes displays statistically significant and consistent differences between two biological states. We identified signaling pathways related to FKBP4 between datasets (low or high FKBP4 expression) through utilizing the GSEA software. In the GSEA software, the main parameters were set as follows: reference gene set: c2.cp.kegg.v6.2.symbols.gmt, the number of genes identifying distinct pathways in each analysis: 1,000, The number of gene set rearrangements in each analysis: 1,000. What's more, the normalized 
126 enrichment score (NES), nominal $p$-value and false discovery rate (FDR) $q$-value were given to denote

127 the importance of associations between gene sets and pathways.

\section{$128 \quad 2.7 \quad$ Statistical analysis}

129 Mann-Whitney U test was implemented to inspect the FKBP4 expression difference between LUAD 130 tissues and non-tumor tissues. Kruskal-Wallis test was utilized to analyse FKBP4 expression 131 differences among groups. $\chi^{2}$ test was applied to assess the correlation between $F K B P 4$ expression and 132 each clinicopathological parameter. Logrank test was employed to compare the survival rate between 133 the high FKBP4 expression group and the low FKBP4 expression group. On basis of the Cox regression 134 model, we carried out univariate and multivariate survival analysis. Among statistical methods in this 135 study, we took $p<0.05$ as the criterion for determining the significance level.

\section{Results}

\section{$137 \quad 3.1 \quad F K B P 4$ is upregulated in LUAD and Pan-Cancer according to online databases}

138 Through analyzing FKBP4 expression data from various cancers in UALCAN, it was watched that 139 FKBP4 expression level was obviously higher in most human cancers compared with the 140 corresponding normal tissues (Figure 1A). Besides, via analyzing FKBP4 mRNA expression from 551 141 samples (497 LUAD samples and 54 non-tumor samples) in the TCGA cohort, we found that LUAD 142 tissues showed significantly higher FKBP4 expression level than normal tissues (Figure 1B). 143 Meanwhile, this result was validated by performing the differential expression analysis for 54 LUAD 144 samples and paired normal samples in the TCGA cohort (Figure 1C), and a CPTAC cohort (111 145 LUAD samples and 111 normal samples) also proved this result (Figure 1D). 
A

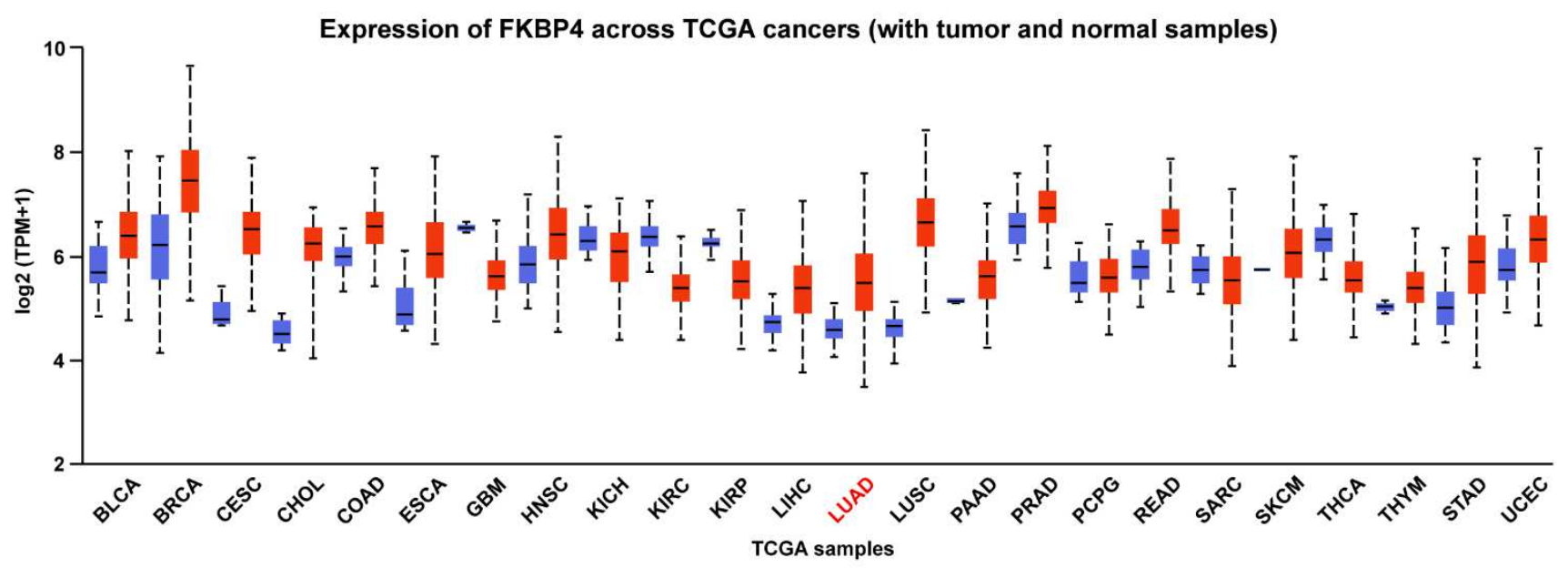

B

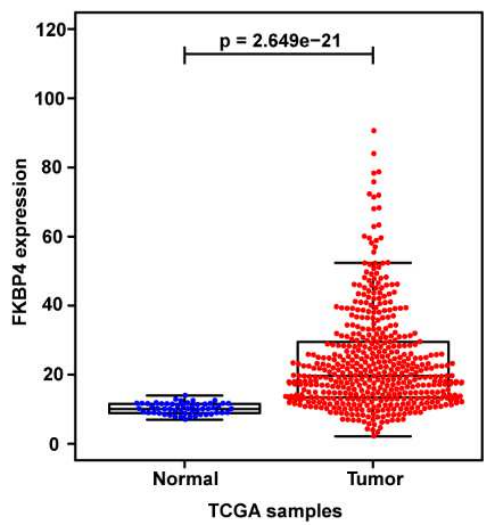

C

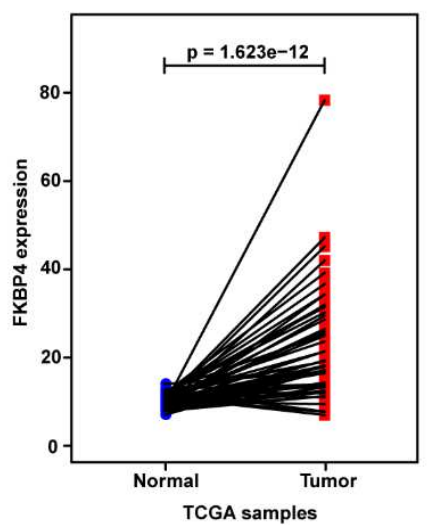

D

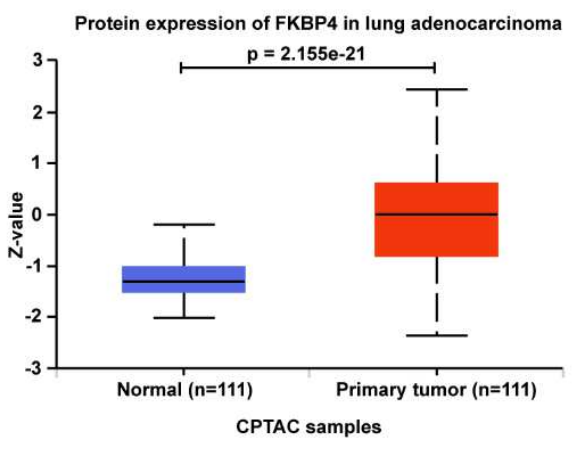

FIGURE 1 | FKBP4 expression in normal human tissues, human tumors, and in LUAD. (A) FKBP4 mRNA expression between multiple human cancers and corresponding normal samples in UALCAN database. (B) FKBP4 mRNA expression between LUAD samples and normal samples from the TCGA cohort. (C) FKBP4 mRNA expression between LUAD samples and paired normal samples from the TCGA cohort. (D) FKBP4 protein expression between LUAD samples and normal samples from the CPTAC cohort.

\subsection{Verification of FKBP4 upregulation in LUAD by TMAs, qRT-PCR and SMD}

153 With the aim of characterizing FKBP4 expression status in LUAD, we first detected FKBP4 protein expression between LUAD samples and normal samples in the human protein atlas database (http://www.proteinatlas.org) (Figure 2A). Then, FKBP4 mRNA expression was validated in a clinical $F K B P 4$ cohort containing 51 pairs of fresh frozen tissue specimens, indicating that FKBP4 was upregulated in LUAD tissues ( $\boldsymbol{p}<\mathbf{0 . 0 0 1}$, Figure 2B). Furthermore, relying on the FKBP4 expression data for LUAD patients from the GEO dataset (Table 2), we performed a comprehensive meta-analysis. As shown in (Figure 2C), according to the random effect model (95\% CI: 0.43-1.20), the combined SMD of $F K B P 4$ was 0.82 . the $I$-square value was $76 \%(p<0.001)$. The above results manifested that $F K B P 4$ was significantly expressed in LUAD. 
A

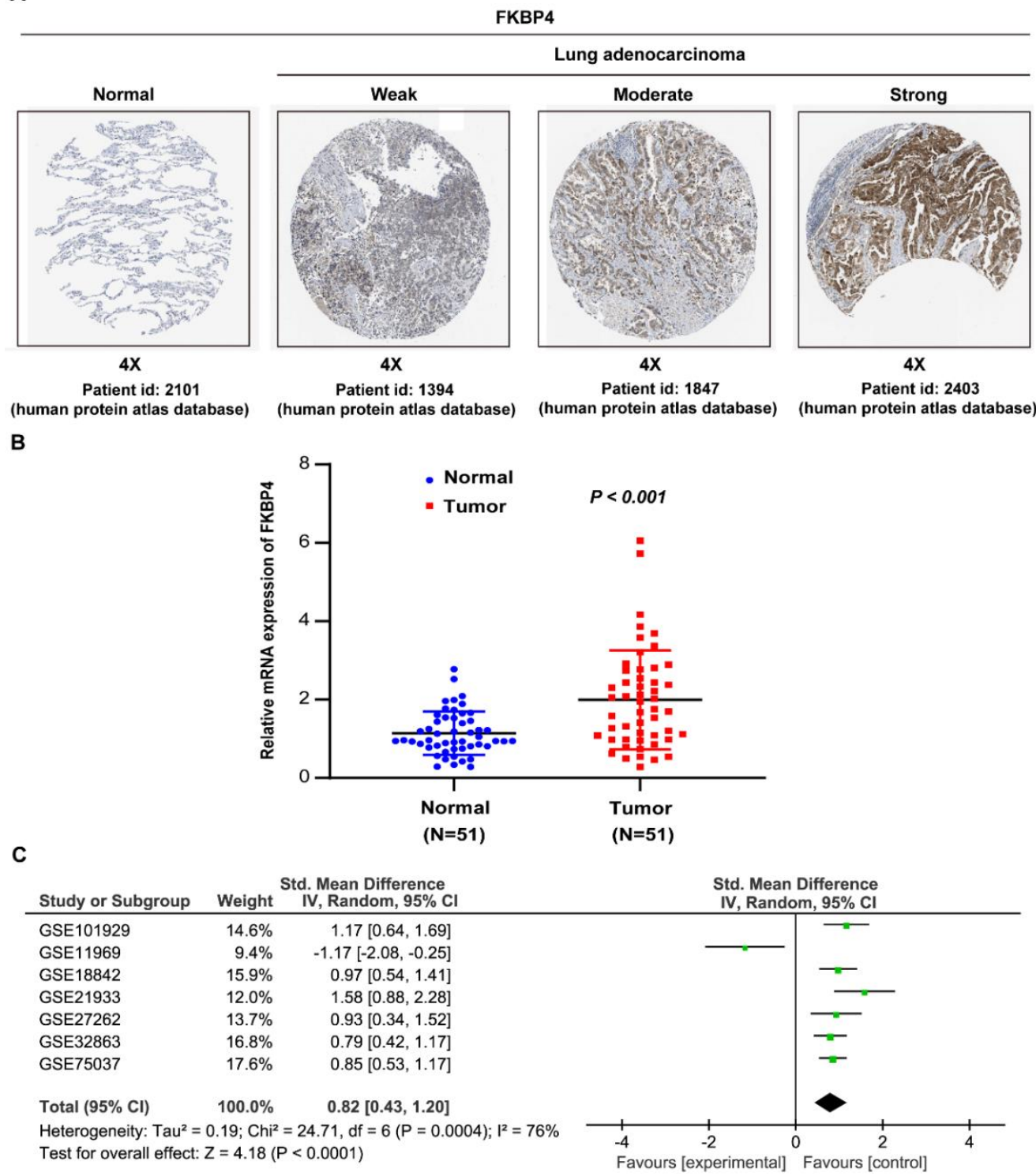

FIGURE $2 \mid$ FKBP4 is upregulated in LUAD patient specimens. (A) Representative images of $F K B P 4$ expression 164 from the human protein atlas (http://www.proteinatlas.org) online database. (B) Expression of $F K B P 4$ between 51 165 LUAD tissues and corresponding non-tumor tissues were detected by qRT-PCR $(p<0.001)$. (C) Forest plot of $F K B P 4$ 166 expression data from GEO microarrays. Gene Expression Omnibus (GEO), standard mean difference (SMD), 167 confidence interval (CI).

\section{$168 \quad 3.3 \quad F K B P 4$ is associated with malignant progression in patients with LUAD}

169 Based on FKBP4 expression and corresponding clinical data from TCGA database, we conducted the 170 in-depth analysis and watched that the expression of $F K B P 4$ was different in groups classified 171 according to gender $(\boldsymbol{p}<\mathbf{0 . 0 0 1}$, Figure 3A), pathological stage $(\boldsymbol{p}=\mathbf{0 . 0 0 3 2}$, Figure 3C), T classification $172(\boldsymbol{p}<0.001$, Figure 3D), N classification $(\boldsymbol{p}=\mathbf{0 . 0 3 1}$, Figure 3E), and $\mathrm{M}$ classification $(\boldsymbol{p}=\mathbf{0 . 0 1 1}$, Figure 173 3F). In addition, depending on the clinical data of 316 LUAD patients from TCGA database, we explored associations between the $F K B P 4$ expression and clinicopathological parameters. As described in Table 3, the high FKBP4 expression level was significantly correlated with gender $(p=0.007)$, pathological stage $(p=0.013)$, T stage $(p=0.014)$, lymph node metastasis $(p=0.003)$ and distant 
upregulated expression of $F K B P 4$ mRNA in LUAD was significantly related to gender $(\mathrm{OR}=1.708$ for male vs. female, $p=0.004)$, pathological stage $(\mathrm{OR}=1.645$ for stage II vs. stage I and $p=0.031$, $\mathrm{OR}=3.329$ for stage IV vs. stage $\mathrm{I}$ and $p=0.009)$, $\mathrm{T}$ classification $(\mathrm{OR}=1.807$ for $\mathrm{T} 2 \mathrm{vs}$. $\mathrm{T} 1$ and $p=0.004$, $\mathrm{OR}=3.098$ for $\mathrm{T} 3$ vs. $\mathrm{T} 1$ and $p=0.002$, $\mathrm{OR}=4.498$ for $\mathrm{T} 4 \mathrm{vs}$. $\mathrm{T} 1$ and $p=0.006$ ), lymph node metastasis $(\mathrm{OR}=1.479$ for Positive vs. Negative and $p=0.047)$ and distant metastasis $(\mathrm{OR}=3.231$ for Yes vs. no 183 and $p=0.015)$.

A

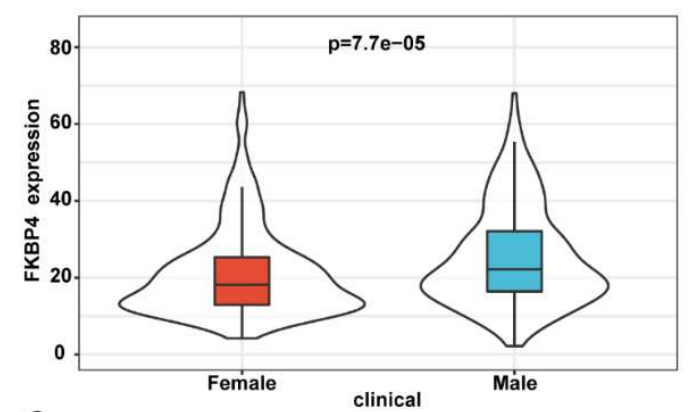

C

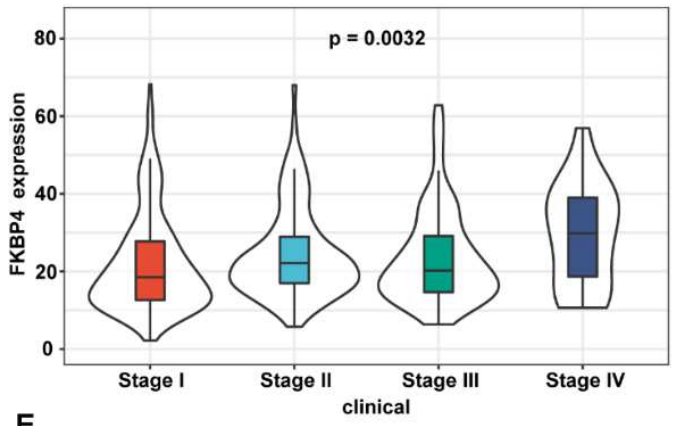

E

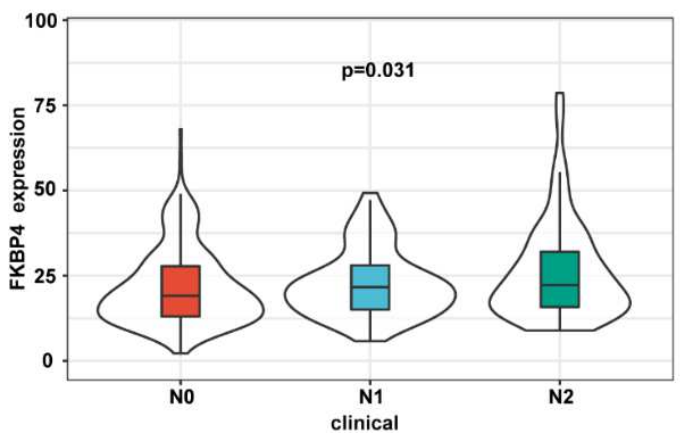

B

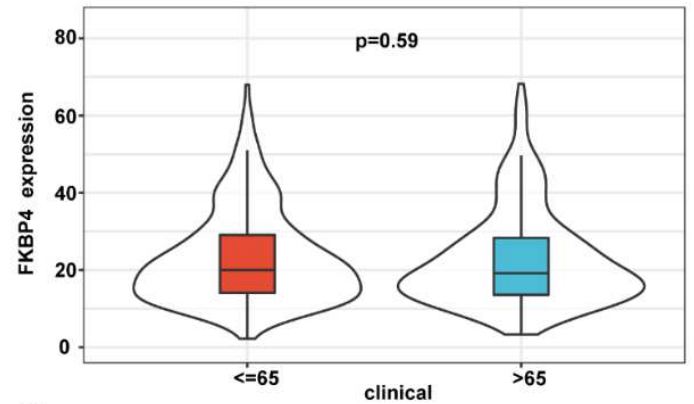

D

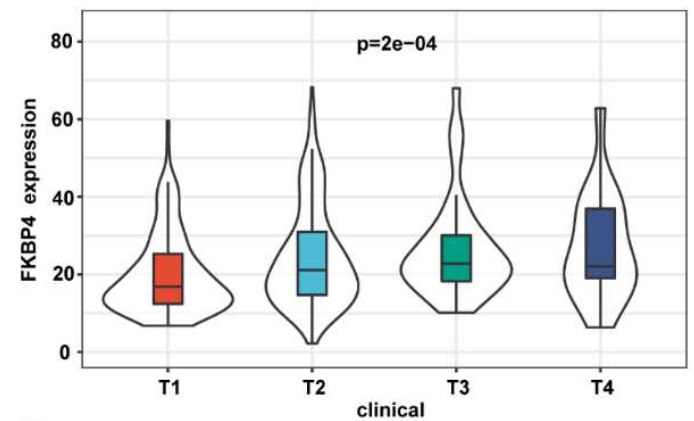

$\mathbf{F}$

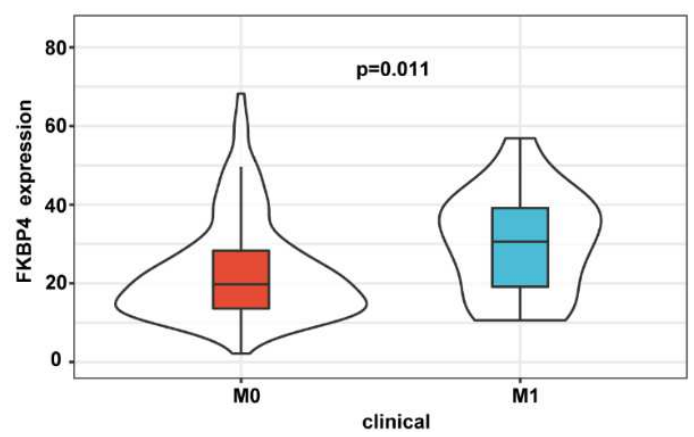

185 FIGURE 3 | Violin plot to evaluate FKBP4 mRNA expression in LUAD patients based on clinical 186 characteristics. (A) Gender. (B) Age. (C) Pathological stage. (D) T classification. (E) N classification. (F) M 187 classification.

188 TABLE 3 | Relationships between FKBP4 expression and clinicopathological parameters in LUAD

\begin{tabular}{|c|c|c|c|c|}
\hline \multirow{2}{*}{$\begin{array}{l}\text { Clinicopathological } \\
\text { parameters }\end{array}$} & \multicolumn{2}{|c|}{ FKBP4 expression } & \multirow[t]{2}{*}{ Total } & \multirow[t]{2}{*}{$p$-value } \\
\hline & High $(n=158)$ & Low $(n=158)$ & & \\
\hline \multicolumn{5}{|l|}{ Age } \\
\hline$<65$ years & $82(53.2)$ & $72(46.8)$ & 154 & 0.260 \\
\hline$\geqslant 65$ years & $76(46.9)$ & $86(53.1)$ & 162 & \\
\hline \multicolumn{5}{|l|}{ Gender } \\
\hline Male & $69(42.6)$ & $93(57.4)$ & 162 & 0.007 \\
\hline
\end{tabular}




\begin{tabular}{llccl}
$\begin{array}{l}\text { Female } \\
\text { Pathological stage }\end{array}$ & $89(57.8)$ & $65(42.2)$ & 154 & \\
$\quad$ I-II & $110(46.0)$ & $129(54.0)$ & 239 & $\mathbf{0 . 0 1 3}$ \\
III-IV & $48(62.3)$ & $29(37.7)$ & 77 & \\
$\begin{array}{l}\text { T classification } \\
\text { T1-T2 }\end{array}$ & $129(47.3)$ & $144(52.7)$ & 273 & $\mathbf{0 . 0 1 4}$ \\
T3-T4 & $29(67.4)$ & $14(32.6)$ & 43 & \\
$\begin{array}{l}\text { Lymph node metastasis } \\
\text { Negative }\end{array}$ & $88(43.8)$ & $113(56.2)$ & 201 & $\mathbf{0 . 0 0 3}$ \\
$\quad$ Positive & $70(60.9)$ & $45(39.1)$ & 115 & \\
$\begin{array}{l}\text { Distant metastasis } \\
\quad \text { No }\end{array}$ & $142(48.1)$ & $153(51.9)$ & 295 & $\mathbf{0 . 0 1 3}$ \\
$\quad 16(76.2)$ & $5(23.8)$ & 21 & \\
\hline
\end{tabular}

189 Bold values indicate $p<0.05$.

190 TABLE 4 | FKBP4 expression correlated with clinicopathological parameters

\begin{tabular}{lccc}
\hline Clinicopathological parameters & Total $(\boldsymbol{N})$ & $\begin{array}{c}\text { Odds ratio in } \\
\text { FKBP4 expression }\end{array}$ & $\boldsymbol{p}$-value \\
\hline $\begin{array}{l}\text { Age } \\
\quad<65 \text { vs. } \geq 65\end{array}$ & 458 & $1.036(0.717-1.498)$ & 0.851 \\
$\begin{array}{l}\text { Gender } \\
\quad \text { Male vs. female }\end{array}$ & 477 & $1.708(1.188-2.461)$ & $\mathbf{0 . 0 0 4}$ \\
$\begin{array}{l}\text { Pathological stage } \\
\quad \text { Stage II vs. stage I }\end{array}$ & 366 & $1.645(1.049-2.592)$ & $\mathbf{0 . 0 3 1}$ \\
$\quad$ Stage III vs. stage I & 335 & $1.591(0.957-2.659)$ & 0.074 \\
$\quad$ Stage IV vs. stage I & 282 & $3.329(1.398-8.818)$ & $\mathbf{0 . 0 0 9}$ \\
$\quad \begin{array}{l}\text { T classification } \\
\quad \text { T2 vs. T1 }\end{array}$ & 414 & $1.807(1.210-2.714)$ & $\mathbf{0 . 0 0 4}$ \\
$\quad$ T3 vs. T1 & 200 & $3.098(1.529-6.516)$ & $\mathbf{0 . 0 0 2}$ \\
$\quad$ T4 vs. T1 & 178 & $4.498(1.632-14.491)$ & $\mathbf{0 . 0 0 6}$ \\
Lymph node metastasis & & & \\
$\quad$ Positive vs. Negative & 465 & $1.479(1.007-2.181)$ & $\mathbf{0 . 0 4 7}$ \\
Distant metastasis & & & \\
$\quad$ Yes vs. no & 348 & $3.231(1.317-9.099)$ & $\mathbf{0 . 0 1 5}$ \\
\hline
\end{tabular}

191 Bold values indicate $p<0.05$.

\section{$192 \quad 3.4 \quad$ High expression of FKBP4 in LUAD patients is related to poor OS}

193 In this study, Kaplan-Meier risk estimate was employed to evaluate the prognostic role of FKBP4 in 194 LUAD. Comparing low FKBP4 expression group with high FKBP4 expression group, we observed 195 that high $F K B P 4$ expression group has more associations with a poor OS (Figure 4B). When taking 196 gender and race into account, we have reached the same result (Figure 4C, Figure 4D). Besides, 197 univariate and multivariate analyses were implemented on 316 LUAD samples in TCGA database to 198 study the impact of FKBP4 expression and clinicopathological factors on survival. Univariate analysis 199 indicated four important predictors of survival including pathological stage (HR: 1.654, 95\% CI: 1.401200 1.951, $p=0.000$ ), T stage (HR: 1.632, 95\% CI: 1.315-2.024, $p=0.000)$, N stage (HR: 1.790, 95\% CI: 201 1.459-2.196, $p=0.000$ ) and FKBP4 expression (HR: 1.012, 95\% CI: 1.005-1.018, $p=0.001$ ) (Table 5). 202 Multivariate analysis demonstrated that the high FKBP4 expression was a vital independent predictor 203 of a poor OS in LUAD (HR: 1.008, 95\% CI: 0.999-1.015, $p=0.012$ ) (Figure 4A, Table 5). 
A

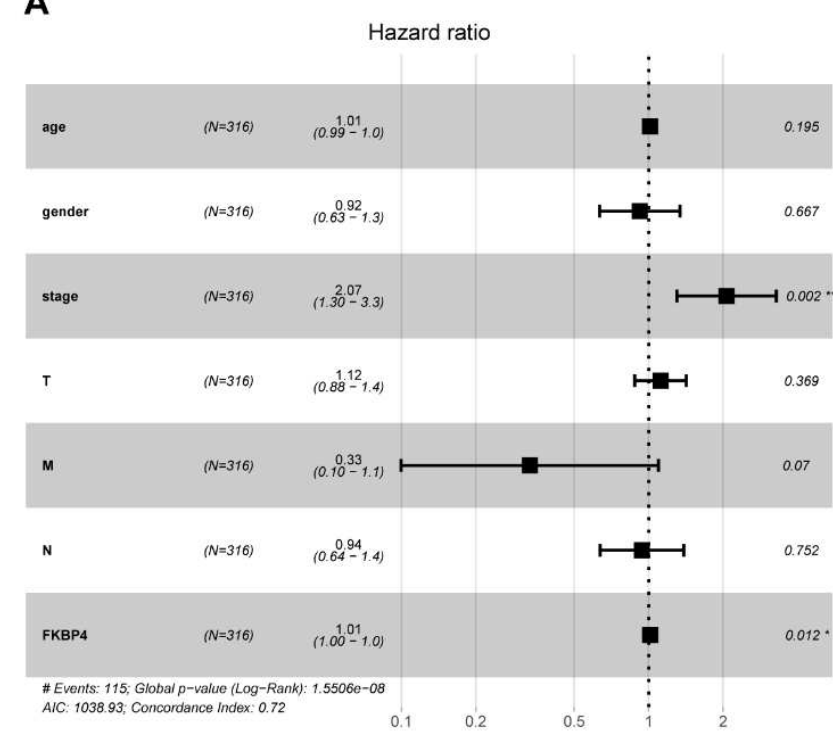

C

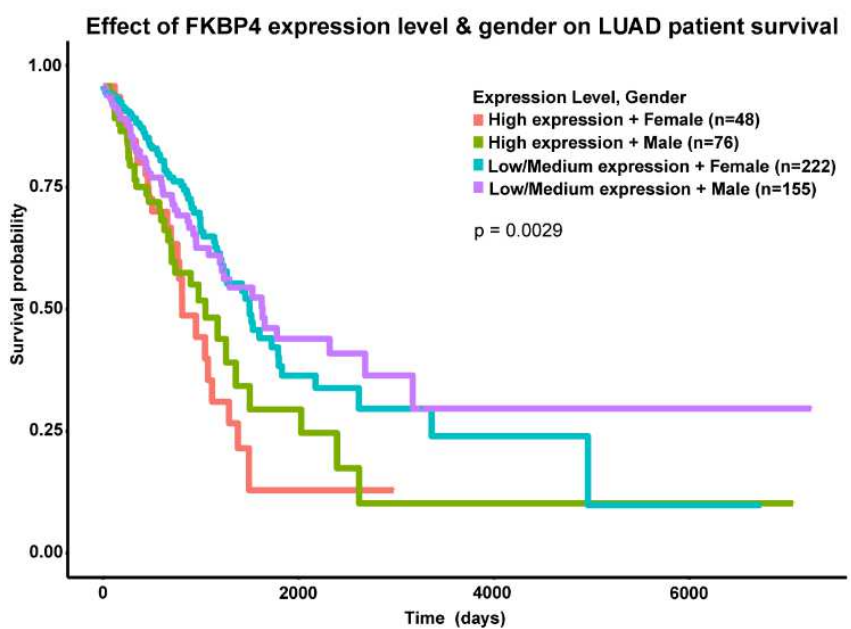

B
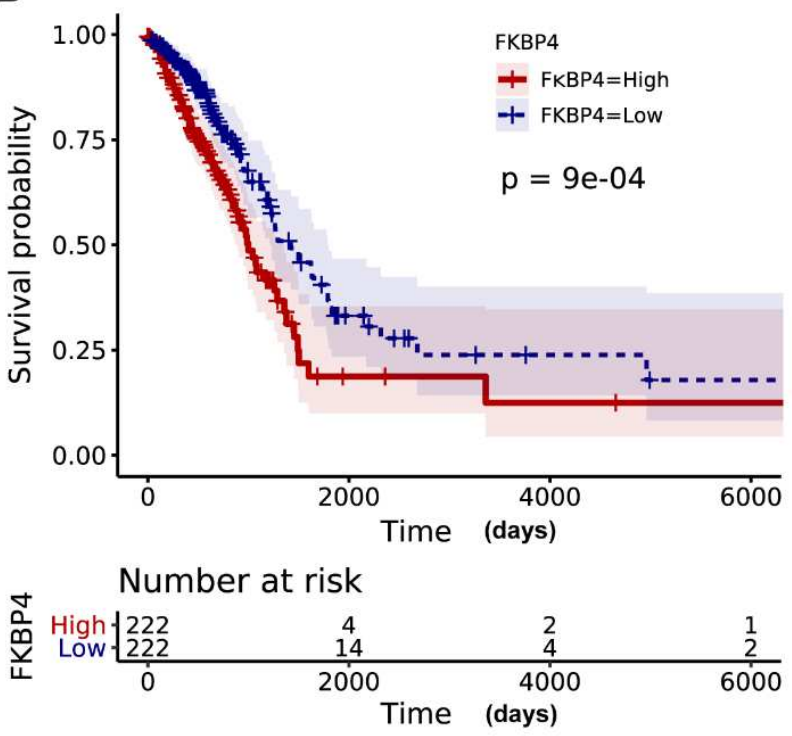

D

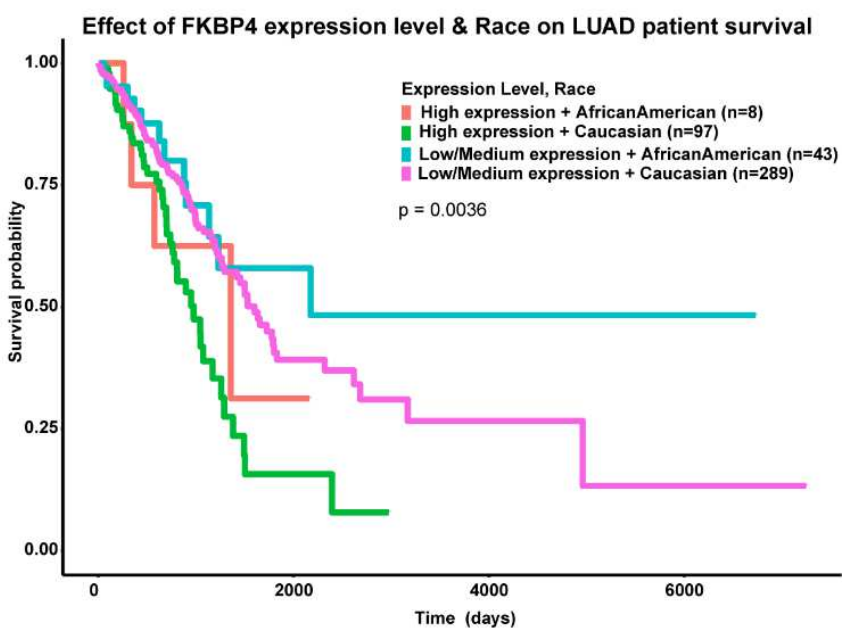

FIGURE 4 | Prognostic role of $\boldsymbol{F K B P 4}$ in patients with LUAD. (A) Forest plot indicated that $F K B P 4$ was an independent predictor of poor survival rate (HR: 1.008, 95\% CI: 0.999-1.015, $p=0.012$ ). (B) Kaplan-Meier curve of the association between FKBP4 mRNA expression and the prognosis of LUAD patients. (C) Effect of FKBP4 expression level and gender on LUAD patient survival. (D) Effect of FKBP4 expression level and race on LUAD patient survival. hazard ratio (HR), confidence interval (CI). $\left({ }^{*} \mathrm{p}<0.05, * * \mathrm{p}<0.01, * * * \mathrm{p}<0.001\right)$.

210 TABLE 5 | Univariate and multivariate analysis of FKBP4 expression correlations among LUAD patients

\begin{tabular}{lccccccc}
\hline Parameter & \multicolumn{3}{c}{ Univariate analysis } & & \multicolumn{3}{c}{ Multivariate analysis } \\
\cline { 2 - 4 } & HR & $\mathbf{9 5 \%}$ CI & $\boldsymbol{p}$ & & HR & $\mathbf{9 5 \%}$ CI & $\boldsymbol{p}$ \\
\hline Age & 1.002 & $0.983-1.021$ & 0.843 & & 1.011 & $0.991-1.031$ & 0.195 \\
Gender & 1.035 & $0.717-1.494$ & 0.852 & & 0.908 & $0.623-1.321$ & 0.667 \\
Pathological stage & 1.654 & $1.401-1.951$ & $\mathbf{0 . 0 0 0}$ & & 1.987 & $1.255-3.144$ & $\mathbf{0 . 0 0 2}$ \\
T & 1.632 & $1.315-2.024$ & $\mathbf{0 . 0 0 0}$ & & 1.160 & $0.912-1.476$ & 0.369 \\
N & 1.790 & $1.459-2.196$ & $\mathbf{0 . 0 0 0}$ & & 0.975 & $0.661-1.439$ & 0.752 \\
M & 1.757 & $0.964-3.203$ & 0.066 & & 0.374 & $0.116-1.208$ & 0.070 \\
FKBP4 & 1.012 & $1.005-1.018$ & $\mathbf{0 . 0 0 1}$ & & 1.008 & $0.999-1.015$ & $\mathbf{0 . 0 1 2}$ \\
\hline
\end{tabular}

211 Bold values indicate $p<0.05$. 
213 Here, we adopted the GSEA to further investigate possible mechanism of $F K B P 4$ in promoting LUAD

214 progression. Specifically, the patients with LUAD were first divided into high or low FKBP4 215 expression groups. Then, according to normalized enrichment score (NES), false discovery rate (FDR) $q$-value and nominal (NOM) $p$-value, fifteen significantly enriched signaling pathways with the high FKBP4 expression phenotype were identified and listed as pyrimidine metabolism, pentose phosphate pathway, cell cycle, RNA polymerase, proteasome, spliceosome, DNA replication, citrate cycle tca cycle, oocyte meiosis, aminoacyl tRNA biosynthesis, fructose and mannose metabolism, base excision repair, RNA degradation, basal transcription factors and protein export (Figure 5A, Table 6). We also gave details of four important signal pathways (Figure 5B-5E).

A
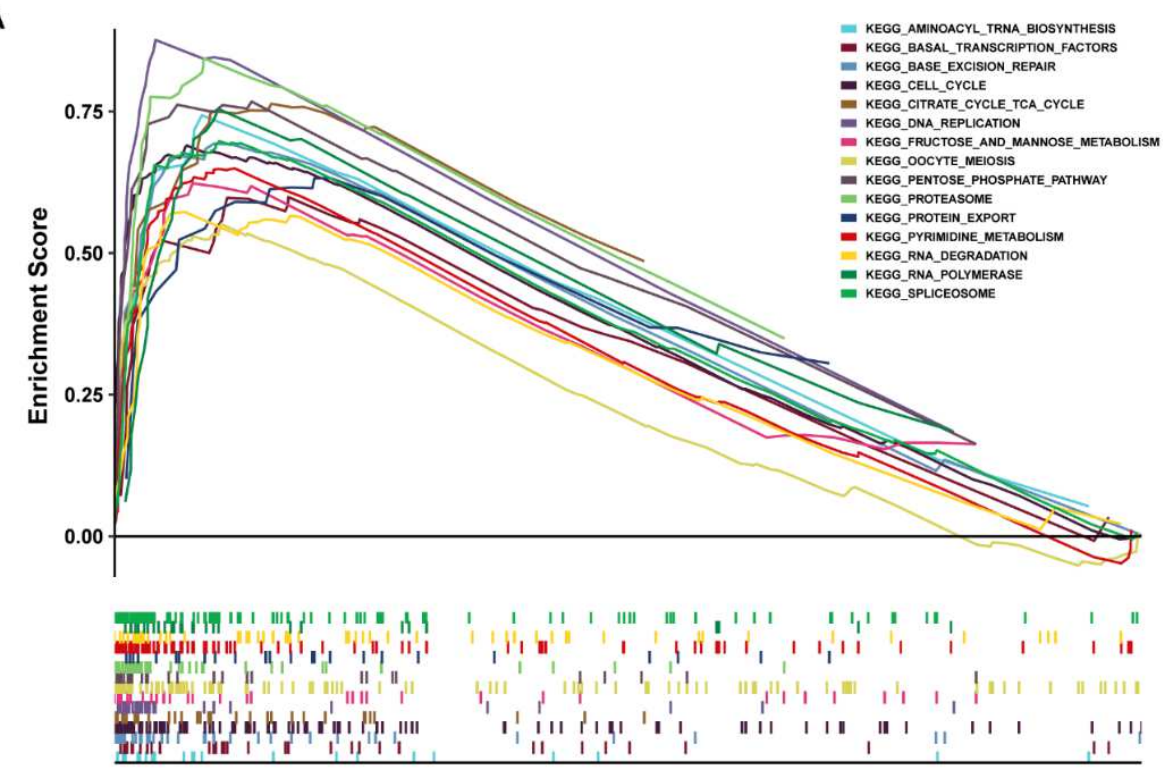

B

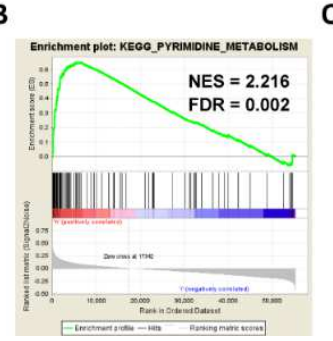

high expression<----------->low expression

C

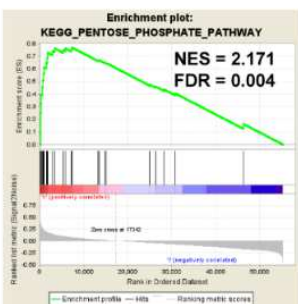

D

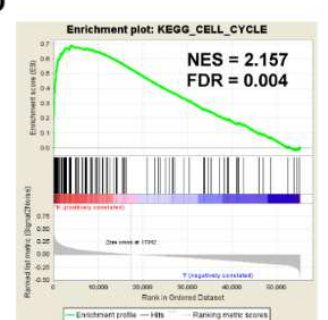

E

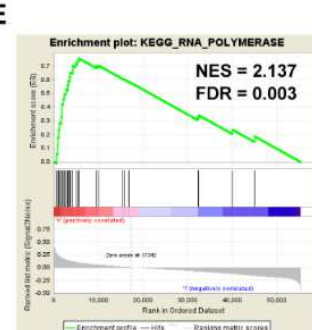

FIGURE 5 | The research on the potential mechanism of $F K B P 4$ in promoting LUAD progression. (A) The merged enrichment plot related to 15 signal pathways. (B) The details of KEGG_PYRIMIDINE_METABOLISM enrichment plot. (C) The details of KEGG_PENTOSE_PHOSPHATE_PATHWAY enrichment plot. (D) The details of KEGG_CELL_CYCLE enrichment plot. (E) The details of KEGG_RNA_POLYMERASE enrichment plot.

227 TABLE 6 | Gene sets enriched in the high FKBP4 expression phenotype

\begin{tabular}{lccc}
\hline Gene set name & NES & NOM $\boldsymbol{p}$-value & FDR $\boldsymbol{q}$-value \\
\hline KEGG_PYRIMIDINE_METABOLISM & 2.216 & 0.000 & 0.002 \\
KEGG_PENTOSE_PHOSPHATE_PATHWAY & 2.171 & 0.000 & 0.004 \\
KEGG_CELL_CYCLE & 2.157 & 0.000 & 0.004 \\
KEGG_RNA_POLYMERASE & 2.137 & 0.000 & 0.003 \\
KEGG_PROTEASOME & 2.099 & 0.000 & 0.005
\end{tabular}




\begin{tabular}{llll} 
KEGG_SPLICEOSOME & 2.079 & 0.002 & 0.006 \\
KEGG_DNA_REPLICATION & 2.024 & 0.000 & 0.012 \\
KEGG_CITRATE_CYCLE_TCA_CYCLE & 2.022 & 0.002 & 0.009 \\
KEGG_OOCYTE_MEIOSIS & 2.022 & 0.002 & 0.009 \\
KEGG_AMINOACYL_TRNA_BIOSYNTHESIS & 1.996 & 0.000 & 0.010 \\
KEGG_FRUCTOSE_AND_MANNOSE_ & 1.977 & 0.000 & 0.010 \\
METABOLISM & & & \\
KEGG_BASE_EXCISION_REPAIR & 1.909 & 0.004 & 0.016 \\
KEGG_RNA_DEGRADATION & 1.881 & 0.008 & 0.020 \\
KEGG_BASAL_TRANSCRIPTION_FACTORS & 1.878 & 0.007 & 0.019 \\
KEGG_PROTEIN_EXPORT & 1.721 & 0.034 & 0.050 \\
\hline
\end{tabular}

228 Normalized enrichment score (NES), nominal (NOM), false discovery rate (FDR).

\section{Discussion}

230 To our best knowledge, more and more researchers are engaged in the research of diagnostic and 231 prognostic markers related to LUAD. As we know, some researchers have claimed that FKBP4 is 232 abnormally expressed in types of cancers. With rapid development of the comprehensive sequencing technology, massive genomic data has been easily available, which has become more feasible for the discovery and verification of biomarkers. In the study, we first analyzed the FKBP4 expression level among various human tumors via utilizing database UALCAN.

In the research, we first adopted a comprehensive analysis method to study the role of $F K B P 4$ expression in LUAD, particularly as a prognostic biomarker for LUAD. Besides, by identifying the signaling pathways associated with FKBP4 in LUAD, we revealed the underlying mechanisms that affect LUAD occurrence and progression. In detail, based on the TCGA cohort, we watched that FKBP4 expression level in LUAD was significantly higher than that in normal tissues. The result was proved by qRT-PCR and public TMAs from the mRNA and protein level, respectively. We also performed meta-analysis on the GEO cohort to detect FKBP4 expression level in LUAD and drew a conclusion consistent with the previous experiments. The result turned out that FKBP4 might play as an oncogene and have a huge impact on LUAD occurrence and progression. Additionally, statistical analysis showed that FKBP4 expression level was different in groups classified by pathological stage, $\mathrm{T}$ stage, $\mathrm{N}$ stage and $\mathrm{M}$ stage. Further analysis indicated that the high FKBP4 expression level was significantly correlated with pathological stage, T classification, lymph node metastasis and distant metastasis. In summarize, the above evidences manifested that FKBP4 expression at the mRNA level is related to some important clinicopathological parameters.

According to Kaplan-Meier survival analysis, we observed that high FKBP4 expression group was worse than that of low $F K B P 4$ expression group. The univariate analysis revealed that pathological stage, $\mathrm{T}$ stage and $\mathrm{N}$ stage were related to the prognosis of LUAD patients, and the high FKBP4 expression was associated with poor OS. To sum up, FKBP4 was expected to be an independent prognostic factor for the OS in LUAD patients.

GSEA method was applied to analyze signaling pathways related to FKBP4 in LUAD. As a result, pyrimidine metabolism, pentose phosphate pathway, cell cycle, RNA polymerase, proteasome, spliceosome, DNA replication, citrate cycle tca cycle, oocyte meiosis, aminoacyl tRNA biosynthesis, fructose and mannose metabolism, base excision repair, RNA degradation, basal transcription factors and protein export were found to be correlated with LUAD progression. Abnormal pyrimidine metabolism can affect tumor invasion and metastasis [11, 12]. Pentose phosphate pathway plays a critical role in regulating cancer cell growth via providing cells with NADPH for detoxification of 
reactive oxygen species, reductive biosynthesis and ribose biosynthesis [13, 14]. Besides, the pentose phosphate pathway is regulated by lots of factors, including tumor suppressors, oncoproteins and intracellular metabolites. Dysregulation of the pentose phosphate pathway flux dramatically impacts cancer growth and survival $[15,16]$. What's more, unscheduled proteolysis of cell cycle regulators leads to tumorigenesis $[17,18]$. Basic transcription factors are necessary for the initiation of RNA polymerase II transcription and can maintain the basic level of transcription. Once the basic level of transcription is imbalanced, it will affect the function of RNAs, and then induce the occurrence and progression of tumors $[19,20]$. Proteasome is a multi-protein organelle that participates in cellular proteostasis through destroying damaged or short-lived proteins. Proteasome is involved in all cell processes including decisions on cell survival or death, cell cycle, and differentiation. These processes are also important in cancer. [21, 22]. Moreover, Novel functions of cytoplasmic aminoacyl-tRNA synthetases may shape the hallmarks of cancer [23, 24].

There is no denying that this research has some limitations. On one hand, the clinical data are incomplete and lack some specific information including surgical treatments and surgical details. On the other hand, due to the limitations of the available data, we can not offer a FKBP4-related specific axis that regulates LUAD occurrence and progression.

\section{Conclusion}

279 In summary, taking full use of FKBP4 expression data related to LUAD patients, we found that the $280 F K B P 4$ expression in LUAD tissues is higher than that in non-tumor tissues. We also watched that the up-regulation of FKBP4 is dramatically correlated with some clinicopathological features of LUAD. Thus, we believed that the up-regulation of $F K B P 4$ promotes the occurrence and progression of LUAD According to univariate and multivariate survival analyses, the increased FKBP4 expression in LUAD was identified as an independent risk factor for shorter OS. To sum up, we hold the opinion that FKBP4 may become a promising biomarker for diagnosis and prognosis of LUAD.

\section{Acknowledgments}

287 Not applicable.

\section{$288 \quad 7 \quad$ Authors' contributions}

289 ST and XT designed the overall idea of this study, conceived the experiments, analyzed the data, 290 prepared the figures and tables and authored the drafts of the paper. SW, PZ, XZ, HH, XH and JL 291 collected the data from the TCGA and GEO dataset, performed the experiments. XT supervised this 292 study and reviewed the drafts of the paper. All the authors read and approved the final draft.

\section{$293 \quad 8 \quad$ Funding}

294 This study was supported by Hunan Province "domestic first-class cultivation discipline" Integrated 295 Traditional Chinese and Western medicine open fund project (Grant No. 2019ZXJH02), Chinese 296 Medicine Scientific Research Program of Hunan Province (Grant No. 2021235) and Changsha 297 Municipal Natural Science Foundation (Grant No. kq2014087).

\section{Availability of data and materials}


Available datasets in this study were analyzed and can be downloaded from The Cancer Genome Atlas

300 (https://portal.gdc.cancer.gov/). The NCBI Gene Expression Omnibus (GSE101929, GSE11969,

301 GSE18842, GSE27262, GSE32863 and GSE75037).

$30210 \quad$ Ethics approval and consent to participate

303 The project was granted approval by the Ethics Committee of the Affiliated Hospital of Xuzhou

304 Medical University.

\section{Consent for publication}

306 All authors consent to publication.

$307 \quad 12$ Competing interests

308 The authors declare no conflict of interest.

30913 Reference

310 1. Bray F, Ferlay J, Soerjomataram I, Siegel RL, Torre LA, Jemal A: Global cancer statistics 311 2018: GLOBOCAN estimates of incidence and mortality worldwide for 36 cancers in 185 countries. CA: a cancer journal for clinicians 2018, 68(6):394-424.

2. Xu JY, Zhang C, Wang X, Zhai L, Ma Y, Mao Y, Qian K, Sun C, Liu Z, Jiang S et al: Integrative Proteomic Characterization of Human Lung Adenocarcinoma. Cell 2020, 182(1):245-261.e217.

3. Herbst RS, Morgensztern D, Boshoff C: The biology and management of non-small cell lung cancer. Nature 2018, 553(7689):446-454.

4. Chen Z, Fillmore CM, Hammerman PS, Kim CF, Wong KK: Non-small-cell lung cancers: a heterogeneous set of diseases. Nature reviews Cancer 2014, 14(8):535-546.

5. Campbell JD, Alexandrov A, Kim J, Wala J, Berger AH, Pedamallu CS, Shukla SA, Guo G, Brooks AN, Murray BA et al: Distinct patterns of somatic genome alterations in lung adenocarcinomas and squamous cell carcinomas. Nature genetics 2016, 48(6):607-616.

6. Devarakonda S, Morgensztern D, Govindan R: Genomic alterations in lung adenocarcinoma. The Lancet Oncology 2015, 16(7):e342-351.

7. Song S, Tan Y: Expression of FKBP52 in the ovaries of PCOS rats. International journal of molecular medicine 2019, 43(2):868-878.

8. Xiong H, Chen Z, Zheng W, Sun J, Fu Q, Teng R, Chen J, Xie S, Wang L, Yu XF et al: FKBP4 is a malignant indicator in luminal A subtype of breast cancer. Journal of Cancer 2020, 11(7):1727-1736.

9. Mangé A, Coyaud E, Desmetz C, Laurent E, Béganton B, Coopman P, Raught B, Solassol J: FKBP4 connects mTORC2 and PI3K to activate the PDK1/Akt-dependent cell proliferation signaling in breast cancer. Theranostics 2019, 9(23):7003-7015. Pathway. Analytical cellular pathology (Amsterdam) 2020, 2020:6021602. 
11. Wang X, Liu R, Zhu W, Chu H, Yu H, Wei P, Wu X, Zhu H, Gao H, Liang J et al: UDPglucose accelerates SNAI1 mRNA decay and impairs lung cancer metastasis. Nature 2019, 571(7763):127-131.

12. Zhou Q, Li H, Li Y, Tan M, Fan S, Cao C, Meng F, Zhu L, Zhao L, Guan MX et al: Inhibiting neddylation modification alters mitochondrial morphology and reprograms energy metabolism in cancer cells. JCI insight 2019, 4(4).

13. Jiang $\mathrm{P}, \mathrm{Du} \mathrm{W}, \mathrm{Wu}$ M: Regulation of the pentose phosphate pathway in cancer. Protein \& cell 2014, 5(8):592-602.

14. Liu X, Olszewski K, Zhang Y, Lim EW, Shi J, Zhang X, Zhang J, Lee H, Koppula P, Lei G et al: Cystine transporter regulation of pentose phosphate pathway dependency and disulfide stress exposes a targetable metabolic vulnerability in cancer. Nature cell biology 2020, 22(4):476-486.

15. Patra KC, Hay N: The pentose phosphate pathway and cancer. Trends in biochemical sciences 2014, 39(8):347-354.

16. Shan C, Lu Z, Li Z, Sheng H, Fan J, Qi Q, Liu S, Zhang S: 4-hydroxyphenylpyruvate dioxygenase promotes lung cancer growth via pentose phosphate pathway (PPP) flux mediated by LKB1-AMPK/HDAC10/G6PD axis. Cell death \& disease 2019, 10(7):525.

17. Bashir T, Pagano M: Aberrant ubiquitin-mediated proteolysis of cell cycle regulatory proteins and oncogenesis. Advances in cancer research 2003, 88:101-144.

18. Kastan MB, Bartek J: Cell-cycle checkpoints and cancer. Nature 2004, 432(7015):316-323.

19. Werner F, Weinzierl RO: Direct modulation of RNA polymerase core functions by basal transcription factors. Molecular and cellular biology 2005, 25(18):8344-8355.

20. Ramsay EP, Abascal-Palacios G, Daiß JL, King H, Gouge J, Pilsl M, Beuron F, Morris E, Gunkel P, Engel C et al: Structure of human RNA polymerase III. Nature communications 2020, 11(1):6409.

21. Voutsadakis IA: Proteasome expression and activity in cancer and cancer stem cells. Tumour biology: the journal of the International Society for Oncodevelopmental Biology and Medicine 2017, 39(3):1010428317692248.

22. Mofers A, Pellegrini P, Linder S, D'Arcy P: Proteasome-associated deubiquitinases and cancer. Cancer metastasis reviews 2017, 36(4):635-653.

23. Wang J, Yang XL: Novel functions of cytoplasmic aminoacyl-tRNA synthetases shaping the hallmarks of cancer. The Enzymes 2020, 48:397-423.

24. Park SG, Schimmel P, Kim S: Aminoacyl tRNA synthetases and their connections to disease. Proceedings of the National Academy of Sciences of the United States of America 2008, 105(32):11043-11049. 